\title{
The impact of photovoltaic systems on power losses and voltage profiles in a real medium voltage distribution network
}

\author{
Nevena Srećković ${ }^{1}$, Gorazd Štumberger ${ }^{1}$ \\ ${ }^{1}$ University of Maribor \\ Faculty of Electrical Engineering and Computer Science \\ Smetanova 17, 2000 Maribor, Slovenia \\ Phone: +3862 220 7056, Fax: +38622525481 \\ e-mail: nevena.sreckovic1@um.si , gorazd.stumberger@um.si
}

\begin{abstract}
Higher penetration of distributed generation units (DG) into the distribution network (DN) has imminent influence on DN's power quality and system losses. With DG units being properly placed and sized, numerous positive effects on DN can be achieved. This paper focuses specifically on influence of active and reactive power generation from photovoltaic (PV) systems on voltage profiles and power losses in DN. Main goal of the paper is to evaluate, if power injections from installed PV systems can contribute to the power loss reduction. A case study on realistic model of medium voltage part of DN in town Maribor has been performed. Sizing and suitable location for PV systems that can be installed in the future has been obtained by assessment of buildings' roof photovoltaic potential. The results presented in the paper show that proper power injections can help reducing power losses in the discussed DN.
\end{abstract}

\section{Key words}

Distribution network, photovoltaic power injection, loss reduction, LiDAR data

\section{Introduction}

With increased interest in implementation of smart gird concept, distribution networks (DN) are changing from passive to active systems. With inevitably higher penetration of distributed generation (DG) units, it is necessary to assess all the possible influences that installed DGs can have on the DN. Among available renewable sources, photovoltaic (PV) is gaining much interest, due to its gradual technology development and cost reduction. Since PV inverters provide maximum power only during short time periods, remaining power capacity available may be used for voltage control, by controlling the reactive power generation [1]. Furthermore, reduction of active power losses can be achieved, by proper local reactive power injections, which can be used in minimization of operating costs [2]. Approach for efficient installation of PV systems, by combining geographical, economic and technical aspects has been presented in [5].
This paper deals with the influence of active and reactive power injection from PV systems on voltage profiles and active power losses in a real medium voltage (MV) DN. Beside existing DG, additional PV units were taken into account by determining the most suitable roofs for future PV installations. The voltage profiles and system losses were determined by load flow calculations. They were compared for different operating conditions.

\section{Assessing photovoltaic potential}

Building - integrated PV systems represent convenient way of integrating DG into the urban areas. Lukač et al. [3] presented efficient method for finding and evaluating suitable buildings' roofs for PV installations, by novel PV potential assessment method. Data were obtained using areal LiDAR scanning, by considering nonlinear efficiency characteristics of PV modules with solar inverters and by long - term solar irradiance measurements made with pyranometer. Roofs' inclinations and shadowing from vegetation were also taken into account. Results of average solar potential values of the roofs in the part of city of Maribor are presented on Fig. 1.

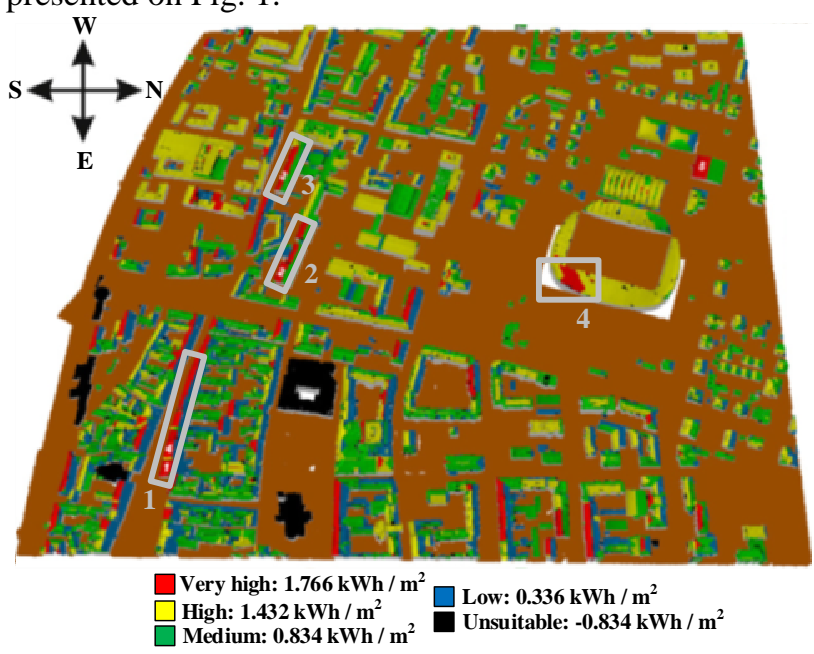


Fig. 1. Visualization of test sites' solar potential ratings [3] Roofs have been categorized by suitability for PV installations using coloring, and most suitable roofs for PV installation, have been marked and numbered.

\section{Discussed distribution network}

MV part of DN in town Maribor (Slovenia) (Fig. 2), during normal operating conditions, consists from two radial distribution networks, which can be looped during extreme conditions. First one is connected to the Koroška Vrata substation (in the future referred to as MVDN A) and the second one is connected to the Melje substation (in the future referred to as MVDN B). One - line diagrams of both models are presented on Fig 3. and Fig

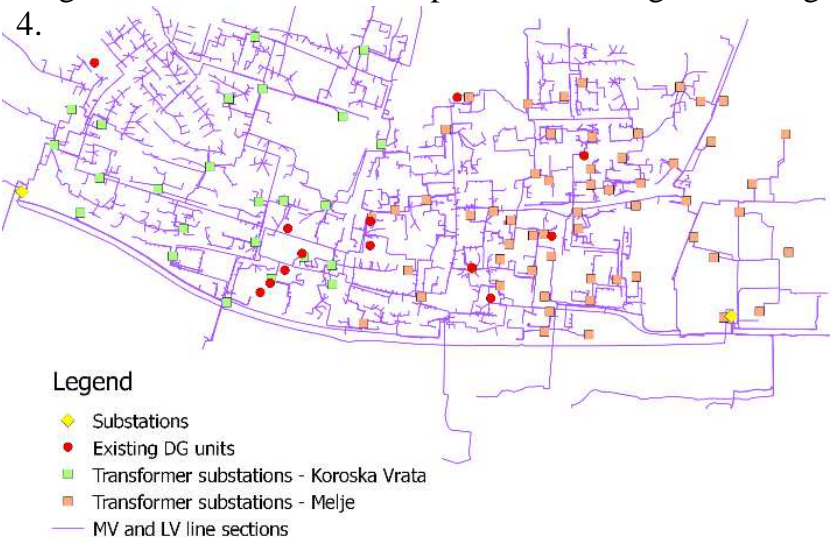

Fig. 2. Part of the distribution network in Maribor

Existing DN has six DG units (four PV systems and two cogenerations) installed in five nodes of MVDN A, as well as the five DG units (one PV system and four cogenerations) in MVDN B. The most suitable roofs for installation of PV systems in the part of the city of Maribor, based on sites' solar potential ratings, have been presented in [3]. Four groups of suitable roofs, marked and numbered in Fig. 1., have been chosen as the most suitable for installation of PV systems discussed in this work.
Available power values of additional PV units $\left(\boldsymbol{P}_{\text {gen,add }}\right)$, have been determined and presented in Table I, and colored red in Figs. 3, 4.

Table I: Power of additional PV units

\begin{tabular}{|c|c|c|}
\hline Group of roofs & $\boldsymbol{P}_{\text {gen,add }}(\mathbf{k W})$ & Connection to: \\
\hline 1 & 234.73 & MVDN B (node 35) \\
\hline 2 & 125.68 & MVDN A (node 26) \\
\hline 3 & 101.10 & MVDN A (node 10) \\
\hline 4 & 88.75 & MVDN A (node 38) \\
\hline
\end{tabular}

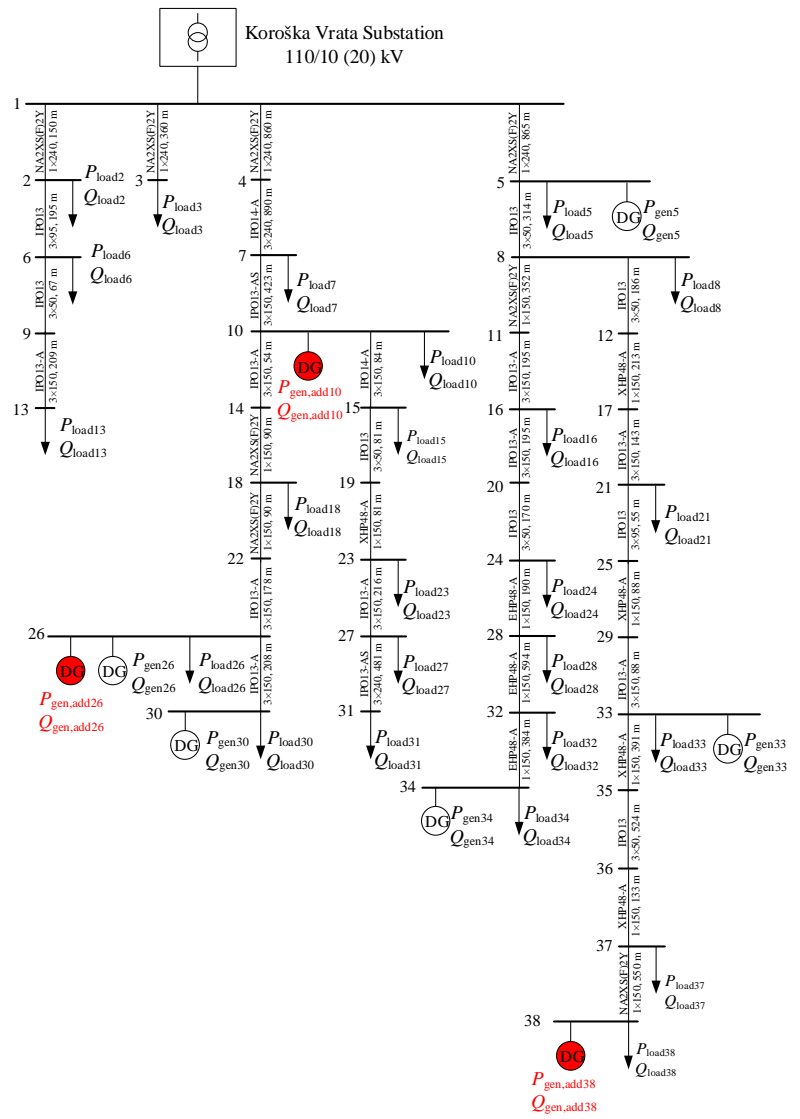

Fig. 3. One - line diagram of MVDN A

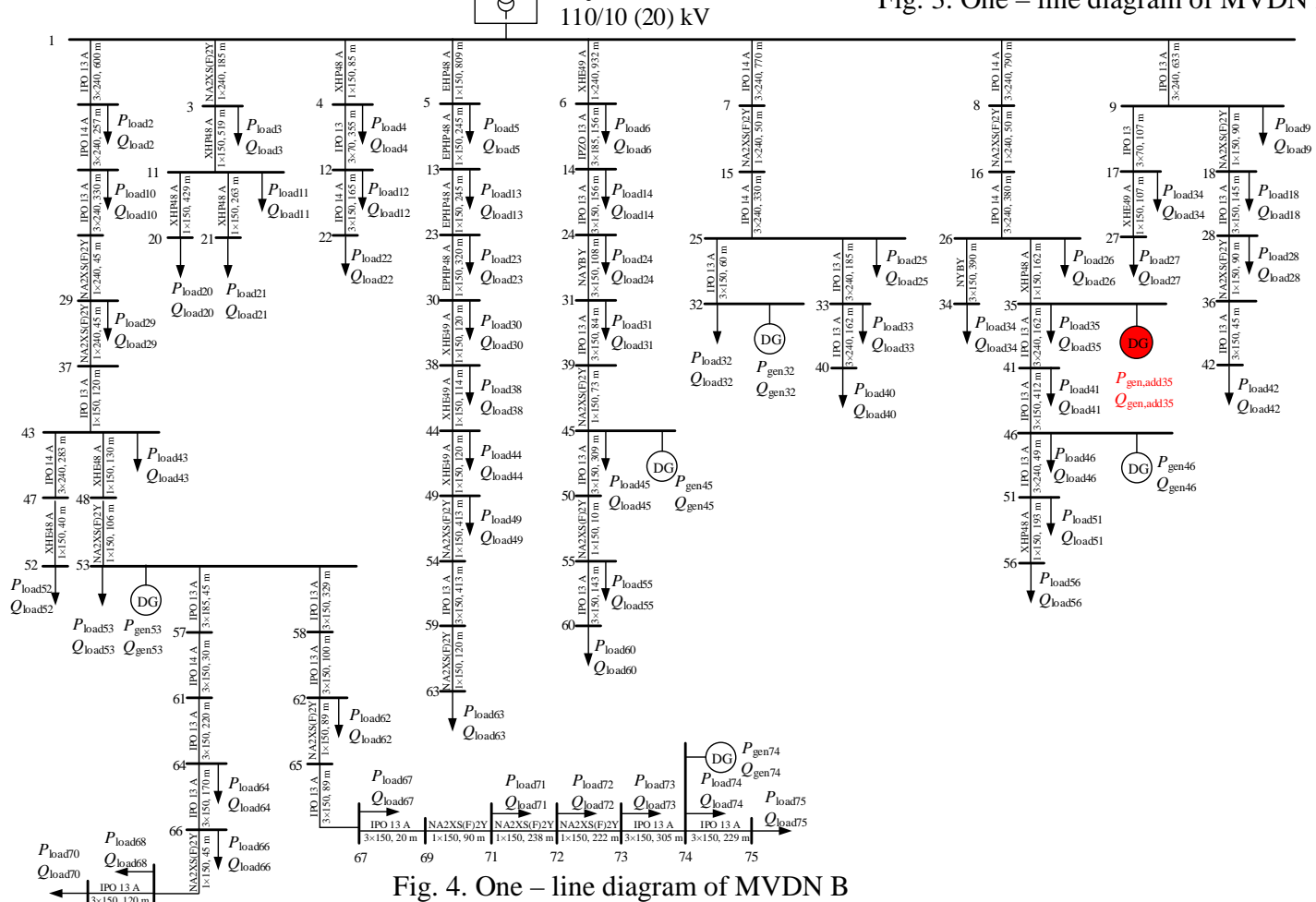




\section{Results}

Voltage profiles and system losses were determined using load flow calculation for both distribution networks MVDN A and MVDN B. Three operating scenarios were discussed and calculations were performed for:

- systems containing only loads,

- $\quad$ systems with already present DG, and both present and additional PV units,

- $\quad$ systems with different reactive power injections from present, and both present and additional PV units.

Single-phase representation of test models is used, and backward - forward sweep method, as presented in [4], has been implemented using Matlab.

It has been shown that proper active and reactive power injections from PV units have positive effects on voltage profile and system losses, as long as the location and the amount of power generation are adjusted to the system requirements.

\section{A. $\quad M V D N A$}

Presented are results, obtained for the first test system.

1) Loading, without generation from $D G$ units Loading factor $x$, used for scaling the maximal power of loads $\left(P_{\text {load }}\right)$, is introduced in order to show how the increase of loading level decreases bus voltages (Fig. 5) and rapidly increases losses (Table II), in the system where no generating units exist.

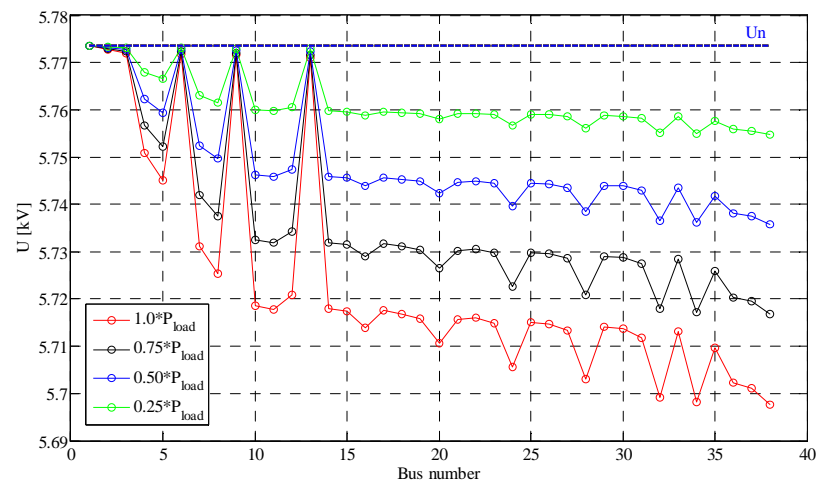

Fig. 5. Voltage profiles for different loading levels in MVDN A without DG units

Table II: Loss comparison in MVDN A for different loading levels and without DG units

\begin{tabular}{|c|c|c|}
\hline Loading factor $x$ & $P_{\text {loss }, \mathrm{x}}(\mathrm{kW})$ & $P_{\text {loss }, \mathrm{x}} / P_{\text {loss }, 1}$ \\
\hline 1.00 & 50.802 & 1 \\
\hline 0.75 & 28.419 & 0.559 \\
\hline 0.50 & 12.562 & 0.247 \\
\hline 0.25 & 3.124 & 0.061 \\
\hline
\end{tabular}

2) Loads, and existing and added DG units present Bus voltages (Fig. 6) and system losses (Table III) of MVDN A system, containing only existing DG unites are compared to the values obtained when three additional PV units are added to the DN. Loading level of $50 \%$ has been taken into account.

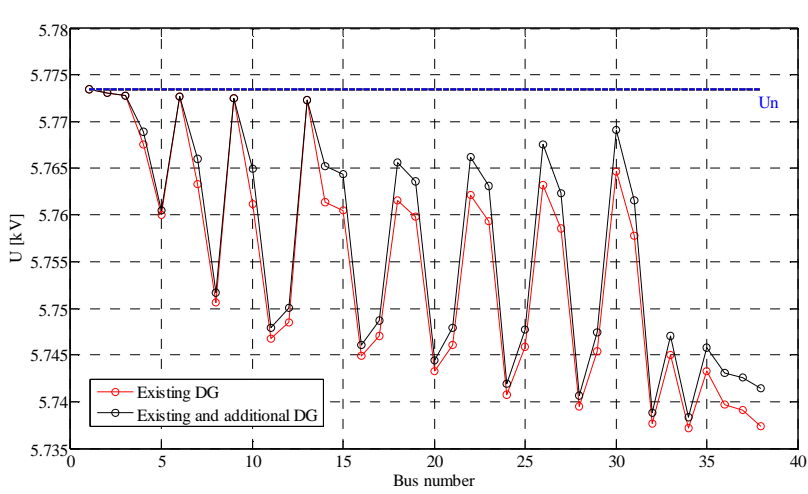

Fig. 6. Voltage profiles in MVDN A with existing and additional DG used

Table III: Loss comparison for different DG units used

\begin{tabular}{|c|c|c|}
\hline & $P_{\text {loss }}(\mathrm{kW})$ & $\begin{array}{c}\text { Loss } \\
\text { reduction }(\%)\end{array}$ \\
\hline No DG units & 12.562 & $/$ \\
\hline Existing DG units & 8.060 & 35.84 \\
\hline Existing and added DG units & 6.997 & 44.30 \\
\hline
\end{tabular}

Obtained values show that DG units already present in the system can help reduce total system losses by more than a third, compared to the system with no DG units. Installation of PV systems on proposed locations, based on assessed solar potential, leads to additional $8 \%$ loss reduction.

\section{3) Existing and added DG units}

Furthermore, the possibility of reducing losses by proper reactive power injections from PV units has been tested. According to laws in Slovenia, all installed PV systems must be able to operate with $\cos \varphi=0.8$. Therefore, the influence of reactive power injected by the PV systems $\left(Q_{\text {gen }}\right)$, has been examined for $Q_{\text {gen }}$ values varying from $-0.75 P_{\text {gen }}$ to $0.75 P_{\text {gen }}$ in steps of $0.25 P_{\text {gen }}$. Scaling factor $q$ is used for changing the value of injected reactive power.

The obtained voltage profiles for loading level of $50 \%$ have been presented in Fig. 7. Obtained loss reduction, compared with the losses when no reactive power is generated, for the cases when existing PV units, and both - existing and added PV units inject different levels of reactive power, are presented in table IV.

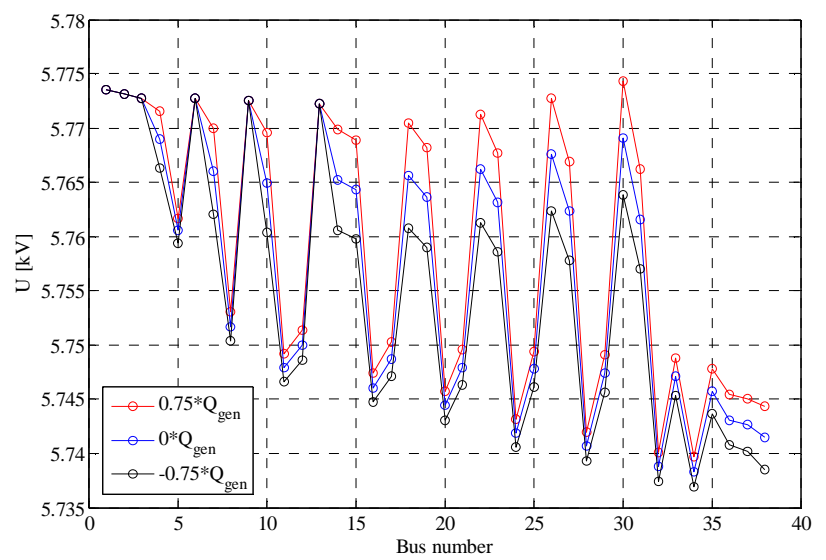


Fig. 7. Voltage profiles in MVDN A with all DG units and loading - variation of reactive power generation

Table IV: Loss comparison for all DG units and loading in MVDN A- variation of reactive power generation

\begin{tabular}{|c|c|c|c|c|}
\hline \multirow{2}{*}{$q$} & \multicolumn{2}{|c|}{$\begin{array}{c}Q_{\text {gen }} \text { from existing } \\
\text { PV units }\end{array}$} & \multicolumn{2}{c|}{$\begin{array}{c}Q_{\text {gen }} \text { from existing and } \\
\text { added PV units }\end{array}$} \\
\cline { 2 - 5 } & $\begin{array}{c}P_{\text {loss,q }} \\
(\mathrm{kW})\end{array}$ & $\begin{array}{c}\text { Loss } \\
\text { reduction }(\%)\end{array}$ & $\begin{array}{c}P_{\text {loss,q }} \\
(\mathrm{kW})\end{array}$ & $\begin{array}{c}\text { Loss } \\
\text { reduction }(\%)\end{array}$ \\
\hline 0.75 & 7.948 & 1.390 & 6.549 & 6.389 \\
\hline 0.50 & 7.983 & 0.955 & 6.647 & 4.989 \\
\hline 0.25 & 8.021 & 0.484 & 6.780 & 3.088 \\
\hline 0 & 8.060 & $/$ & 6.996 & $/$ \\
\hline-0.25 & 8.101 & -0.509 & 7.247 & -3.588 \\
\hline-0.50 & 8.144 & -1.042 & 7.549 & -7.905 \\
\hline-0.75 & 8.188 & -1.588 & 7.902 & -12.950 \\
\hline
\end{tabular}

Negative value of loss reduction implies the rise of the system losses. This is caused by inductive nature of generated reactive power, which causes higher deviations in voltage profile as well. On the other hand - with reactive power having capacitive nature, improvement of voltage profile and reduction of system losses are achieved.

\section{B. $\quad M V D N B$}

Similar analysis as for the test system MVDN A has been performed also for the test system MVDN B. Voltage profiles and losses were determined for different operating conditions. System losses are presented in Tables V - VII and obtained voltage profiles are shown in Figs. $8-10$.

\section{1) Loading, without generation from $D G$ units}

Increase of loading level in MVDN B causes greater system losses (Table V) and greater voltage drops (Fig. 8) than in MVDN A.

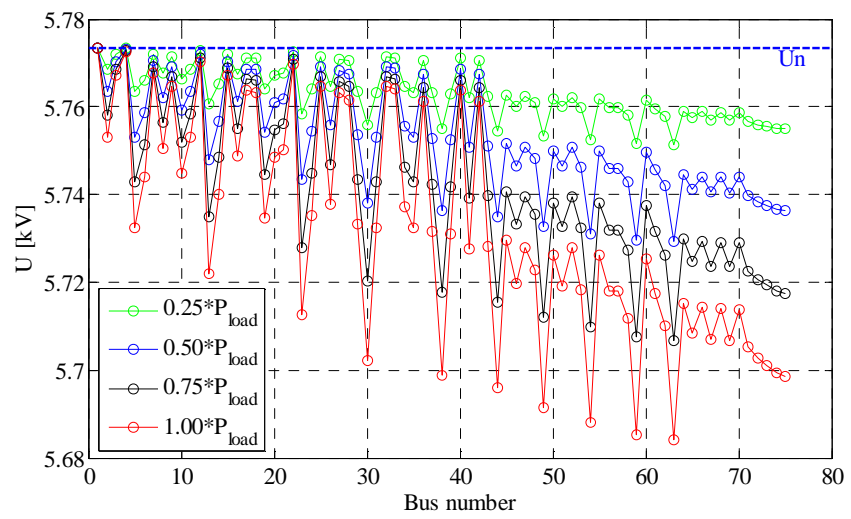

Fig. 8. Voltage profiles in MVDN B for different loading levels and without DG units

Table V: Loss comparison in MVDN B for different loading levels and without DG units

\begin{tabular}{|c|c|c|}
\hline Loading factor $x$ & $P_{\text {loss }, \mathrm{x}}(\mathrm{kW})$ & $P_{\text {loss }, \mathrm{x}} / P_{\text {loss }, 1}$ \\
\hline 1.00 & 120.880 & 1 \\
\hline 0.75 & 67.667 & 0.560 \\
\hline 0.50 & 29.930 & 0.248 \\
\hline 0.25 & 7.447 & 0.062 \\
\hline
\end{tabular}

2) Loads and existing and added DG units present The consideration of the existing DG and additional PV units in the MVDN B, results in reduction of system losses presented in Table VI.

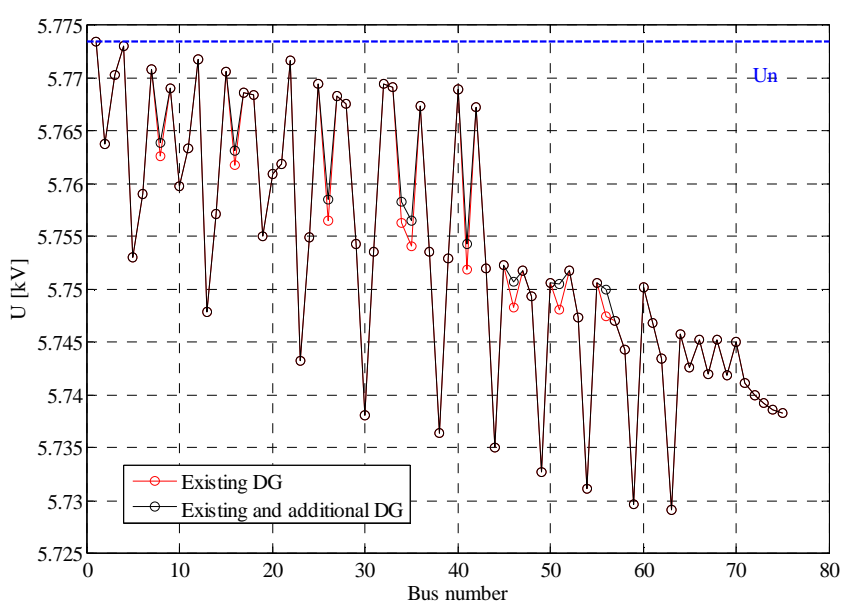

Fig. 9. Voltage profiles in MVDN B with existing and additional DG used

Table VI: Loss comparison in MVDN B for different DG units used

\begin{tabular}{|c|c|c|}
\hline & $P_{\text {loss }}(\mathrm{kW})$ & $\begin{array}{c}\text { Loss } \\
\text { reduction }(\%)\end{array}$ \\
\hline No DG units & 29.930 & $/$ \\
\hline Existing DG units & 28.019 & 6.387 \\
\hline Existing and added DG units & 26.862 & 10.251 \\
\hline
\end{tabular}

\section{3) Existing and added DG units}

Using PV units for generation of reactive power of capacitive nature $(q>0)$ causes improvement of voltage profiles (Fig. 10) and reduction of system losses presented in Table VII. Loading level of $50 \%$ has been used in the simulations.

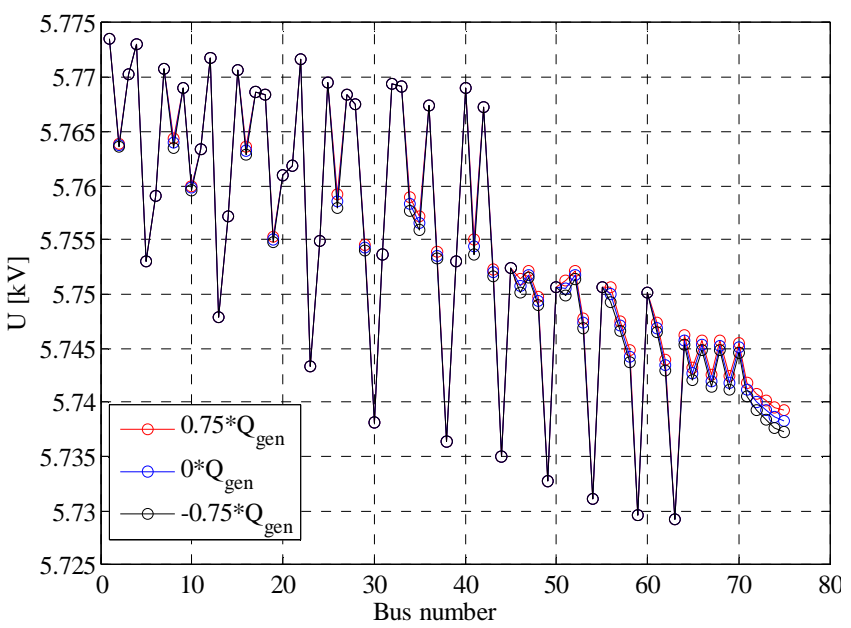

Fig. 10. Voltage profiles in MVDN B with all DG units and loading - variation of reactive power generation 
Table VII: Loss comparison in MVDN B with all DG units and loading - variation of reactive power generation

\begin{tabular}{|c|c|c|c|c|}
\hline & \multicolumn{2}{|c|}{$\begin{array}{c}Q_{\text {gen }} \text { from existing PV } \\
\text { units }\end{array}$} & \multicolumn{2}{c|}{$\begin{array}{c}Q_{\text {gen }} \text { from existing and } \\
\text { added PV units }\end{array}$} \\
\hline$q$ & $\begin{array}{c}P_{\text {loss,q }} \\
(\mathrm{kW})\end{array}$ & $\begin{array}{c}\text { Loss } \\
\text { reduction } \\
(\%)\end{array}$ & $\begin{array}{c}P_{\text {loss, }} \\
(\mathrm{kW})\end{array}$ & $\begin{array}{c}\text { Loss } \\
\text { reduction } \\
(\%)\end{array}$ \\
\hline 0.75 & 27.932 & 0.311 & 26.801 & 0.227 \\
\hline 0.50 & 27.959 & 0.214 & 26.796 & 0.246 \\
\hline 0.25 & 27.988 & 0.111 & 26.816 & 0.171 \\
\hline 0 & 28.019 & 0.000 & 26.862 & 0.000 \\
\hline-0.25 & 28.051 & -0.114 & 26.934 & -0.268 \\
\hline-0.50 & 28.084 & -0.232 & 27.033 & -0.637 \\
\hline-0.75 & 28.119 & -0.357 & 27.157 & -1.098 \\
\hline
\end{tabular}

The loss reduction achieved with generation of reactive power from existing and additional PV units is smaller in the case of $q=0.75$ than in the case of $q=0.50$. This implies that having large amounts of reactive power injected into the single point of the system can cause additional system losses.

\section{Conclusion}

The best locations for the future PV installation have been chosen from assessment of roofs' solar potential [3]. Four groups of roofs having highest values of solar irradiance were chosen as possible locations for future PV installations and were used for presented case study. The aim of this study was to evaluate the impact of existing and additional DG units on voltage profiles and DN losses. The results have shown that higher the penetration of DG units is, greater are benefits on system operation. Proper reactive power generation in PV systems can improve voltage profiles and reduce active power losses.
However, it must be pointed out that the amounts and locations of reactive power injected must be adjusted to fit actual conditions in the DN. The reactive power injection that does not fit the DN requirements, normally leads to the loss increase.

\section{Acknowledgment}

This work was supported in part by Slovenian Research Agency under research contract L2-5489.

\section{References}

[1] S. Weckx, C. Gonzalez, J. Driesen. "Combined Central and Local Active and Reactive Power Control of PV Inverters," IEEE Transactions on Sustainable Energy, Vol. 5, No. 3, pp. 776-784, 2014.

[2] M. Kolenc, I. Papič, B. Blažič. "Coordinated reactive power control to achieve minimal operating costs," International Journal of Electrical Power \& Energy Systems, Vol. 63, pp.1000-1007, 2014.

[3] N. Lukač, D. Žlaus, S. Seme, B. Žalik, G. Štumberger. "Rating of roofs' surfaces regarding their solar potential and suitability for PV systems, based on LiDAR data." Applied energy, Vol. 102, pp. 803-812, 2012.

[4] D. Thukaram, H. M. Wijekoon Banda, J. Jerome. "A robust three phase power flow algorithm for radial distribution systems." Electric Power System Research, Vol. 50, pp. 227-236, 1999.

[5] S. Kucuksari, A. M. Khaleghi, M. Hamidi, Y. Zhang, F. Szidarovszky, G. Bayraksan, Y. J. Son. "An Integrated GIS, optimization and simulation framework for optimal PV size and location in campus area environments." Applied Energy. Vol. 113, pp. 1601 - 1613, 2014. 